Voix et Images

voixetimages

\title{
Le Pays renversé de Denys Delâge et l'Identité usurpée de Jean Morisset
}

\section{Andrée Mercier}

Volume 11, numéro 3 (33), printemps 1986

Yolande Villemaire

URI : https://id.erudit.org/iderudit/200590ar

DOI : https://doi.org/10.7202/200590ar

Aller au sommaire du numéro

Éditeur(s)

Université du Québec à Montréal

ISSN

0318-9201 (imprimé)

1705-933X (numérique)

Découvrir la revue

Citer cet article

Mercier, A. (1986). Le Pays renversé de Denys Delâge et l'Identité usurpée de Jean Morisset. Voix et Images, 11(3), 551-554. https://doi.org/10.7202/200590ar d'utilisation que vous pouvez consulter en ligne.

https://apropos.erudit.org/fr/usagers/politique-dutilisation/ 


\title{
Le Pays renversé de Denys Delâge et l'Identité usurpée de Jean Morisset
}

\author{
par Andrée Mercier, Université du Québec à Montréal
}

\section{Le Pays renversé de Denys Delâge ${ }^{1}$}

Denys Delâge n'a pas écrit ici une "Histoire de l'Amérique du nord-est" où seraient répertoriés tous les événements et les moindres acteurs ayant façonné la période 1600-1664. Son but est de décrire le renversement d'un pays et surtout de l'expliquer dans une perspective économique: la conquête de l'Amérique du nord-est vue comme le processus d'annexion de cet espace à l'économie atlantique (D., p. 9).

Ce point de vue, que l'on pourrait craindre réducteur, exige justement de ne plus considérer l'unique formation des sociétés européennes en Amérique, mais de reconnaître d'abord la présence autochtone sur le continent et ce, au delà de l'habituelle introduction à la "préhistoire amérindienne». Delâge présente donc la terre d'Amérique, sa faune et sa flore, ses habitants et leur organisation sociale en période historique: peu à peu se dessine un espace culturel et géographique où existe déjà la spécialisation de l'activité économique des tribus, où circulent humains et marchandises. Toutefois, la description de la société huronne et de son fonctionnement succède, trop rapidement peut-être, à ce vaste panorama. La Huronie servira de modèle privilégié à l'évaluation de l'impact du commerce dès fourrures, vu l'importance des sources et des études qui lui sont consacrées.

Comme l'ensemble des sociétés iroquoiennes, la société huronne repose sur un système de parenté matrilinéaire et matrilocal dont l'unité de base est le clan. Mais Delâge insiste davantage sur une autre clé fondamentale de l'univers social huron: la règle du don qui, en plus d'éliminer les transactions commerciales au sein du clan et même de la communauté, intervient lors des rites de guérison, fonde un code de générosité et d'hospitalité, et surtout porte une grande signification symbolique et religieuse. C'est là qu'apparaît le principal enjeu de l'ouvrage: l'altération rapide de la règle du don conduit au renversement d'un pays entraîné dans la mécanique implacable d'une économie de marché. Plus précisément, le système d'échange inégal entretenu par les puissances colonisatrices bouleverse, de façon définitive, les sociétés amérindiennes. Delâge montre très bien comment l'inégalité des valeurs échangées détériore tous les niveaux d'organisation sociale: le rapport à la 
nature, l'autarcie amérindienne, les guerres intertribales, le temps de travail et de loisir, le contrôle des carrefours de commerce, le niveau de vie.

L' :nalyse de l'auteur s'ajustera d'ailleurs sur le "grand angulaire» de l'économie-monde pour mettre en place le savoir indispensable à l'interprétation de l'histoire coloniale: l'Europe du XVII' siècle, les rivalités interimpérialistes qui l'animent, la conjoncture économique de ses principaux pays, plus spécifiquement les modes de production des puissances de l'époque et la transition de l'Europe du nord-ouest vers un modèle capitaliste. De ce cadre, se détachent les trois centres colonisateurs concurrents en Amérique du nordest: l'Angleterre, la France et surtout la Hollande qui détient l'hégémonie sur le marché mondial au début du siècle (D., p. 22). Sans aucun doute, la reconnaissance de trois modes différents de colonisation vient compléter la description du bouleversement amérindien: la Hollande et ses rapports commerciaux avec les Mohawks (tribu de la Confédération iroquoise) fournit un modèle de relation absolument distinct du pacte Huronie/France. Il faut ici constater la pertinence d'une étude de l'Amérique du nord-est, perspective élargie qui ajoute des paramètres indispensables à la compréhension de la Nouvelle-France même.

Le choix judicieux d'une analyse économique de la période 1600-1664 n'a pas à être démontré: l'histoire coloniale est avant tout affaire de richesses et, déjà, les relations commerciales entre Européens et Autochtones avaient précédé sa véritable mise en place. La valeur certaine de l'ouvrage consiste principalement à avoir su ménager une part égale à la description de l'économie amérindienne, trop souvent occultée au profit des puissances coloniales. Mais la qualité de l'étude réside aussi dans la prise en compte d'autres éléments de transformation: les épidémies qui hypothéquèrent durement la structure et l'avenir des sociétés autochtones (en fauchant très fort dans le groupe des 15-40 ans), de même que la spécificité des modèles colonisateurs. Ainsi, la symbiose unique entre les intérêts marchands et missionnaires (D., p. 129) distingue la stratégie française de colonisation (double heurt pour la Huronie). Pareille offensive idéologique étant absente du comptoir hollandais, les alliés Iroquois seront exempts de ses effets éminemment perturbateurs et conserveront plus longtemps leurs caractéristiques culturelles et religieuses. Quant à 1'Angleterre, c'est une démographie galopante qui, pour l'essentiel, prépare déjà sa suprématie future.

On peut présumer que, désormais, le Pays renversé de Denys Delâge constituera un des outils importants pour ceux que préoccupe l'histoire de l'Amérique du nord au XVII' siècle.

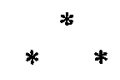




\section{L'Identité usurpée de Jean Morisset ${ }^{2}$}

Avec cet essai de Jean Morisset, nous nous retrouvons maintenant en plein Québec contemporain. Le Français, conquérant parmi d'autres, a cédé depuis belle lurette la place au Canayen conquis. L'Angleterre, que la décennie 1660 laissait au seuil de la suprématie démographique, a définitivement gagné le territoire de l'Amérique du Nord aux dépens des puissances hollandaise et française. Mais elle a aussi subi le morcellement de ses immenses possessions face aux désirs d'autonomie de colonies récalcitrantes.

Si l'on croit reconnaître là les inévitables prémisses au discours nostalgique et amer sur le Québec conquis, on trouvera mieux. Car Jean Morisset propose une voie originale à l'essai «nationaliste».

Tout d'abord, plutôt que de s'associer aux prétentions de la nation québécoise, l'auteur revendique l'identité canadienne que les British North Americans, non contents de s'approprier un pays, devaient aussi usurper. Rapt national donc, si bien réussi, que le véritable Canadien n'aura d'autres ressources que de se créer une identité toute fictive: celle de Québécois; nom emprunté d'ailleurs de Québec, désignation administrative dont les Anglais nous affublèrent après la Conquête (M., p. XVI). Premier indice de notre totale aliénation, le langage doit, au risque même de la confusion, être réhabilité: le Canadien désigne désormais exclusivement le Créole francophone né en Amérique canadienne et son assimilation au Sauvage du Canada (M., p. 44). Tels sont, en raccourci bien sûr, les fondements de l'identité canadienne.

Ce postulat initial s'accompagne d'un autre point de vue original: la nécessité d'une perspective panaméricaine. Ainsi, Jean Morisset dégage le volet complémentaire aux analyses, combien nombreuses, d'un Québec vu de l'intérieur ou dans ses relations avec l'Europe. Il s'agit cette fois de dénoncer, non pas le camouflage de l'identité linguistique, mais celui de la continuité géographique du Québec. Pa reille opération consiste premièrement à contrebalancer le poids des livres écrits en anglais sur le Canádo-Québec (M., p. 13) — et la déformation du fait américain qu'ils entraînent - en écrivant, à notre tour, sur les conquérants. Selon cette nouvelle perspective, l'Amérique du Nord Britannique ne pourra que révéler sa recherche d'une personnalité boréale fondée. tout comme les États-Unis, sur l'élimination des siècles d'histoire non britannique, celle de l'Indien et du Métis, et sur la conquête de l'Ouest. Une réflexion panaméricaine participe d'ailleurs à une interrogation beaucoup plus vaste pour mettre à jour l'ordre continental d'un pays fabriqué sur une Américanité écartée, aussi bien les Francos que les autres groupes minoritaires. Morisset montre bien que la question de l'identité canadienne fait entièrement éclater toute l'historiographie reçue et construite pour légitimer les Anglo-Américains et remet en cause l'Ordre de la Conquête en Nord-Amérique (M., p. 63). Il faut en outre accorder ce crédit à l'auteur: il ne limite pas le point de vue américain aux seuls États-Unis mais intègre encore, sinon privilégie, le paramètre «Amérique Latine» dans l'auto-analyse du Canadien. Cette autre Amérique présente, elle aussi, une image hybride 


\section{VOIX \& IMAGES/33, printemps 1986}

officielle, assujettie à l'Occident industriel et une image cachée tout aussi hybride, moitié-aborigène/moitié-créole (M., p. 25).

L'affaire de la Baie-James constituera pourtant la preuve flagrante de deux siècles d'histoire d'une décolonisation avortée (M., p. 63). "Pierre angulaire" de l'essai, le syndrome de la Baie-James, s'étonne Morisset, est passé à peu près inaperçu chez les intellectuels et les littéraires de la ChoseQuébec (M., p. XVIII). Il faut dire qu'après la lecture de l'analyse fine et lucide qu'il en fera, on s'en étonne aussi.

La Baie-James représente d'abord pour le Québec un processus d'affirmation économique réussie. D'autre part, l'élaboration de la Convention de la Baie-James reproduit toute l'histoire des modalités de la Conquête du Canada (M., p 127): le génocide, sous couvert administratif, du fait indigène et, une fois de plus, l'assimilation du Québec à une tradition idéologique et juridique essentiellement britannico-américaine (M., p 143). Écrite d'abord en anglais (pour ensuite être traduite en français) la Convention de la Baie-James exprime par "la voix de McGill» l'aliénation nationale d'un peuple qui n'a pas voulu savoir ce qu'il faisait et ce qu'on faisait en son nom: l'incarcération zoologique d'un autre peuple (M., p 145). Dans sa quête passionnante de notre identité, Morisset refusera donc à l'événement historique de la Baie-James toute prétention au véritable mythe fondateur du Québec. La sûreté de sa critique, le ton parfois polémique de ses propos nous convainquent de la nécessité de reconnaître, nous aussi, l'Amérique canadienne.

1. Denys Delâge, le Pays renversé. Amérindiens et européens en Amérique du nord-est 1600-1664. Montréal, Éd. Boréal Express, 1985, $424 \mathrm{p}$. Nous référerons désormais à l'ouvrage de D. Delâge de la façon suivante: (D., p.x)

2. Jean Morisset, l'Identité usurpée, Montréal, Éd. Nouvelle Optique, 1985, 158 p., V. I: l'Amérique écartée (Coll. "Matériaux"). À paraître V. 2: la Rédemption nordique et V. 3: la Fabrication du Québec contemporain. Les références à l'ouvrage: (M., p. x). 